\title{
THE POINTWISE STABILITIES OF PIECEWISE LINEAR FINITE ELEMENT METHOD ON NON-OBTUSE TETRAHEDRAL MESHES OF NONCONVEX POLYHEDRA
}

\author{
HUADONG GAO AND WEIFENG QIU
}

\begin{abstract}
Let $\Omega$ be a Lipschitz polyhedral (can be nonconvex) domain in $\mathbb{R}^{3}$, and $V_{h}$ denotes the finite element space of continuous piecewise linear polynomials. On non-obtuse quasi-uniform tetrahedral meshes, we prove that the finite element projection $R_{h} u$ of $u \in$ $H^{1}(\Omega) \cap C(\bar{\Omega})$ (with $R_{h} u$ interpolating $u$ at the boundary nodes) satisfies
\end{abstract}

$$
\left\|R_{h} u\right\|_{L^{\infty}(\Omega)} \leq C|\log h|\|u\|_{L^{\infty}(\Omega)} .
$$

If we further assume $u \in W^{1, \infty}(\Omega)$, then

$$
\left\|R_{h} u\right\|_{W^{1, \infty}(\Omega)} \leq C|\log h|\|u\|_{W^{1, \infty}(\Omega)} .
$$

\section{INTRODUCTION}

In this paper we consider the the Ritz projection $R_{h} u \in V_{r, h}$ of $u \in H^{1}(\Omega) \cap C(\bar{\Omega})$ satisfying

$$
\left(\nabla R_{h} u, \nabla v_{h}\right)_{\Omega}=\left(\nabla u, \nabla v_{h}\right)_{\Omega}, \quad \forall v_{h} \in V_{r, h}^{0},
$$

where $V_{r, h}$ is the finite element subspace of $H^{1}(\Omega)$ composed of piecewise polynomials of degree $r(r \geq 1), V_{r, h}^{0}=H_{0}^{1}(\Omega) \cap V_{r, h}$, and $R_{h} u$ interpolates $u$ at the boundary nodes on $\partial \Omega$. In fact, $R_{h} u$ is the finite element projection of $u$ onto $V_{r, h}$ for the model problem

$$
\Delta u=f \text { in } \Omega \text {, }
$$

with Dirichlet boundary condition on $\partial \Omega$.

Our motivation is to establish the stability in $L^{\infty}(\Omega)$

$$
\begin{aligned}
& \left\|R_{h} u\right\|_{L^{\infty}(\Omega)} \leq C\|u\|_{L^{\infty}(\Omega)}, \\
& \text { or }\left\|R_{h} u\right\|_{L^{\infty}(\Omega)} \leq C|\log h|\|u\|_{L^{\infty}(\Omega)} ;
\end{aligned}
$$

and the stability in $W^{1, \infty}(\Omega)$ (if $u \in W^{1, \infty}(\Omega)$ )

$$
\begin{aligned}
& \left\|R_{h} u\right\|_{W^{1, \infty}(\Omega)} \leq C\|u\|_{W^{1, \infty}(\Omega)}, \\
& \text { or }\left\|R_{h} u\right\|_{W^{1, \infty}(\Omega)} \leq C|\log h|\|u\|_{W^{1, \infty}(\Omega)} .
\end{aligned}
$$

There are a lot of important works for estimates (1.3) and (1.4). [9] and [16] are the first contributions for general quasi-uniform meshes. On convex polygonal domains, [9] considered piecewise linear $(r=1)$ approximation while [16] treated the finite element approximation (for any $r \geq 1$ ) to Neumann problem of (1.2). [13] proved (1.3) and (1.4) on polygonal (can be nonconvex) domains. When $r=1$, the estimates provided in [13] are (1.3b) and (1.4b). Thus, estimates (1.3) and (1.4) are valid for most practical domains in $\mathbb{R}^{2}$. On the contrast, in three dimensional space, all existing works [1, 3, 4, 17, 8, 11, 12, 14, 15] for estimates

2000 Mathematics Subject Classification. 65N30, 65L12.

Key words and phrases. The stability in $L^{\infty}$ and $W^{1, \infty}$, finite element method, nonconvex polyhedra.

The second author is the corresponding author. 
(1.3) and (1.4) are available on either domains with smooth boundary or convex polyhedral domains (Instead of explicit assumptions on domains, [1] needs $\|w\|_{W^{2, p}(\Omega)} \leq C\|\Delta w\|_{L^{p}(\Omega)}$ for some $p>3$ in three dimensional space, for any function $w$ with zero trace on $\partial \Omega$ ).

In this paper, we prove that if the meshes are non-obtuse (all internal dihedral angles of all tetrahedral elements are less than or equal to $\left.\frac{\pi}{2}\right)$, then estimates (1.3b) and (1.4b) hold for the finite element projection (1.1) with piecewise linear finite element space $V_{h}=V_{1, h}$ $(r=1)$.

In Section 2, we provide the main results and all assumptions. In Section 3, we show the proofs of our main results.

\section{MAin RESUlts}

Let $\Omega$ be a Lipschitz polyhedra (can be nonconvex) in $\mathbb{R}^{3}$. We denote by $\mathcal{T}_{h}$ quasi-uniform conforming tetrahedral meshes of $\Omega$. We define $V_{h}=H^{1}(\Omega) \cap P_{1}\left(\mathcal{T}_{h}\right)$ and $V_{h}^{0}=H_{0}^{1}(\Omega) \cap V_{h}$. For any $u \in H^{1}(\Omega)$, we introduce the Ritz projection $R_{h} u \in V_{h}$ to satisfy

$$
\left(\nabla R_{h} u, \nabla v_{h}\right)_{\Omega}=\left(\nabla u, \nabla v_{h}\right)_{\Omega}, \quad \forall v_{h} \in V_{h}^{0},
$$

where $R_{h} u$ interpolates $u$ at the boundary nodes on $\partial \Omega$. In fact, $R_{h} u$ is the finite element projection of $u$ onto $V_{h}$, and (2.1) is exactly the finite element projection (1.1) with $r=1$.

Assumption 2.1. For any $T \in \mathcal{T}_{h}$, all internal dihedral angles of the tetrahedral element $T$ are less than or equal to $\frac{\pi}{2}$. $\mathcal{T}_{h}$ is called non-obtuse tetrahedral meshes of $\Omega$.

Assumption 2.2. The mesh $\mathcal{T}_{h}$ of $\Omega$ can be extended to a larger convex domain $\tilde{\Omega}$ quasiuniformly with $\Omega \Subset \tilde{\Omega}$. We denote by $\tilde{\mathcal{T}}_{h}$ the extension of $\mathcal{T}_{h}$ on $\tilde{\Omega}$.

Remark 2.1. We don't require $\tilde{\mathfrak{T}}_{h}$ introduced in Assumption 2.2 to be non-obtuse for all tetrahedral elements. Only elements $T \in \mathcal{T}_{h}$ need to be non-obtuse.

Theorem 2.2. If Assumption (2.1) and Assumption (2.2) hold, then there is a positive constant $C$ such that for any $u \in H^{1}(\Omega) \cap C(\bar{\Omega})$,

$$
\left\|R_{h} u\right\|_{L^{\infty}(\Omega)} \leq C|\log h|\|u\|_{L^{\infty}(\Omega)} .
$$

Theorem 2.3. If Assumption (2.1) and Assumption (2.2) hold, then there is a positive constant $C$ such that for any $u \in W^{1, \infty}(\Omega)$,

$$
\left\|R_{h} u\right\|_{W^{1, \infty}(\Omega)} \leq C|\log h|\|u\|_{W^{1, \infty}(\Omega)} .
$$

\section{ANALYSis}

Proof. (Proof of Theorem 2.2) Since $u \in C(\bar{\Omega})$, we denote by $\tilde{u}$ the extension of $u$ to $\tilde{\Omega}$, such that $u \in C_{0}(\tilde{\Omega})$ and $\|\tilde{u}\|_{L^{\infty}(\tilde{\Omega})}=\|u\|_{L^{\infty}(\Omega)}$. The existence of $\tilde{u}$ satisfying the above two properties follows from the facts that $u \in C(\bar{\Omega})$ and the Whitney type extension operator $\mathcal{E}_{0}$ in Section 2.2 of Chapter 6 in [17] (see (8) and the proposition in Section 2.2 of Chapter 6 in [17]). We would like to emphasize that we don't need $\tilde{u} \in H^{1}(\tilde{\Omega})$.

We define $\tilde{V}_{h}^{0}=H_{0}^{1}(\tilde{\Omega}) \cap P_{1}\left(\tilde{\mathcal{T}}_{h}\right)$. Let $\tilde{u}_{h} \in \tilde{V}_{h}^{0}$ satisfy

$$
\left(\nabla \tilde{u}_{h}, \nabla \tilde{v}_{h}\right)_{\tilde{\Omega}}=\Sigma_{T \in \tilde{T}_{h}}\left(-\left(\tilde{u}, \Delta \tilde{v}_{h}\right)_{T}+\left\langle\tilde{u}, \nabla \tilde{v}_{h} \cdot \vec{n}\right\rangle_{\partial T}\right), \quad \forall \tilde{v}_{h} \in \tilde{V}_{h}^{0} .
$$


Here $\vec{n}$ is the outward unit normal vector along $\partial T$ for any $T \in \mathcal{T}_{h}$. For any $v_{h} \in V_{h}^{0}=$ $H_{0}^{1}(\Omega) \cap P_{1}\left(\mathcal{T}_{h}\right)$, we denote by $\tilde{v}_{h} \in \tilde{V}_{h}^{0}$ the zero extension of $v_{h}$ to $\tilde{\Omega}$. By (3.1) and the definition of $\tilde{v}_{h}$, it is easy to see that

$$
\begin{aligned}
& \left(\nabla \tilde{u}_{h}, \nabla v_{h}\right)_{\Omega}=\left(\nabla \tilde{u}_{h}, \nabla \tilde{v}_{h}\right)_{\tilde{\Omega}} \\
= & \Sigma_{T \in \tilde{T}_{h}}\left(-\left(\tilde{u}, \Delta \tilde{v}_{h}\right)_{T}+\left\langle\tilde{u}, \nabla \tilde{v}_{h} \cdot \vec{n}\right\rangle_{\partial T}\right) \\
= & \Sigma_{T \in \mathcal{T}_{h}}\left(-\left(\tilde{u}, \Delta \tilde{v}_{h}\right)_{T}+\left\langle\tilde{u}, \nabla \tilde{v}_{h} \cdot \vec{n}\right\rangle_{\partial T}\right) \\
= & \Sigma_{T \in \mathcal{T}_{h}}\left(-\left(u, \Delta v_{h}\right)_{T}+\left\langle u, \nabla v_{h} \cdot \vec{n}\right\rangle_{\partial T}\right)=\left(\nabla u, \nabla v_{h}\right)_{\Omega} .
\end{aligned}
$$

The last equality holds since $u \in H^{1}(\Omega)$. On the other hand, since $\tilde{\Omega}$ is convex and $\tilde{u} \in C_{0}(\tilde{\Omega})$, (3.1) and [8, Theorem 12] imply that

$$
\left\|\tilde{u}_{h}\right\|_{L^{\infty}(\tilde{\Omega})} \leq C|\log h|\|\tilde{u}\|_{L^{\infty}(\tilde{\Omega})}=C|\log h|\|u\|_{L^{\infty}(\Omega)} .
$$

We notice that $R_{h} u \in V_{h}=H^{1}(\Omega) \cap P_{1}\left(\mathcal{T}_{h}\right)$ satisfies

$$
\left(\nabla R_{h} u, \nabla v_{h}\right)_{\Omega}=\left(\nabla u, \nabla v_{h}\right)_{\Omega}, \quad \forall v_{h} \in V_{h}^{0}=H_{0}^{1}(\Omega) \cap V_{h} .
$$

Thus, by the above equation and (3.2), we have that $\left.\left(R_{h} u-\tilde{u}_{h}\right)\right|_{\Omega} \in V_{h}$ and

$$
\left(\nabla\left(R_{h} u-\tilde{u}_{h}\right), \nabla v_{h}\right)_{\Omega}=0, \quad \forall v_{h} \in V_{h}^{0}=H_{0}^{1}(\Omega) \cap V_{h} .
$$

By Assumption (2.1) and [18, Theorem 3.2 and Lemma 5.1(iii)] (or by [2, 5, 6]), the above equation implies that

$$
\left\|R_{h} u-\tilde{u}_{h}\right\|_{L^{\infty}(\Omega)} \leq\left\|R_{h} u-\tilde{u}_{h}\right\|_{L^{\infty}(\partial \Omega)} \leq\|u\|_{L^{\infty}(\partial \Omega)}+\left\|\tilde{u}_{h}\right\|_{L^{\infty}(\partial \Omega)} .
$$

Thus, by (3.3) and (3.4), it is easy to see that

$$
\begin{aligned}
& \left\|R_{h} u\right\|_{L^{\infty}(\Omega)} \leq\left\|R_{h} u-\tilde{u}_{h}\right\|_{L^{\infty}(\Omega)}+\left\|\tilde{u}_{h}\right\|_{L^{\infty}(\Omega)} \\
\leq & \|u\|_{L^{\infty}(\partial \Omega)}+\left\|\tilde{u}_{h}\right\|_{L^{\infty}(\partial \Omega)}+\left\|\tilde{u}_{h}\right\|_{L^{\infty}(\Omega)} \\
\leq & \|u\|_{L^{\infty}(\Omega)}+2\left\|\tilde{u}_{h}\right\|_{L^{\infty}(\Omega)} \leq C|\log h|\|u\|_{L^{\infty}(\Omega)} .
\end{aligned}
$$

The proof is complete.

Proof. (Proof of Theorem 2.3) We denote by $I_{h} u$ the standard interpolation of $u$ on $V_{h}=$ $H^{1}(\Omega) \cap P_{1}\left(\mathcal{T}_{h}\right)$.

By applying Theorem 2.2 to $u-I_{h} u$, we have

$$
\left\|R_{h} u-I_{h} u\right\|_{L^{\infty}(\Omega)} \leq C|\log h|\left\|u-I_{h} u\right\|_{L^{\infty}(\Omega)} .
$$

By inverse inequality and approximation properties of $I_{h}$,

$$
\begin{gathered}
\left\|R_{h} u\right\|_{W^{1, \infty}(\Omega)} \leq\left\|R_{h} u-I_{h} u\right\|_{W^{1, \infty}(\Omega)}+\left\|I_{h} u\right\|_{W^{1, \infty}(\Omega)} \\
\leq C h^{-1}\left\|R_{h} u-I_{h} u\right\|_{L^{\infty}(\Omega)}+C\|u\|_{W^{1, \infty}(\Omega)} \leq C\|u\|_{W^{1, \infty}(\Omega)} .
\end{gathered}
$$

The proof is complete. 


\section{Declarations}

Funding: Huadong Gao is partially supported by National Natural Science Foundation of China under grant number 11871234. Weifeng Qiu is supported by a grant from the Research Grants Council of the Hong Kong Special Administrative Region, China (Project No. CityU 11302718).

The Conflict of Interest Statement: No conflict of interest exists.

Availability of data and material: Not applicable.

Code availability: Not applicable.

Authors' contributions: Huadong Gao and Weifeng Qiu have participated sufficiently in the work to take public responsibility for the content, including participation in the concept, method, analysis and writing. All authors certify that this material or similar material has not been and will not be submitted to or published in any other publication.

\section{REFERENCES}

[1] S. Brenner and L. Scott, The Mathematical Theory of Finite Element Methods, Springer, New York, 2002.

[2] P.G Ciarlet and P.A. Raviart, Maximum principle and uniform convergence for the finite element method, Comput. Methods Appl. Mech. Engrg., 2:17-31, 1973.

[3] A. Demlow, D. Leykekhman, A.H. Schatz and L.B. Wahlbin, Best approximation property in the $W_{\infty}^{1}$ norm on graded meshes, Math. Comp. 81:743-764, 2012.

[4] J. Guzmán, D. Leykekhman, J. Rossmann and A.H. Schatz, Hölder estimates for Green's functions on convex polyhedral domains and their applications to finite element methods, Numer. Math., 112:221-243, 2009.

[5] S. Korotov, M. Kř́žek and P. Neittaanmäki, Weakened acute type condition for tetrahedral triangulations and the discrete maximum principle, Math. Comp., 70(233):107-119, 2000.

[6] M. Kř́žzek and L. Qun, On diagonal dominance of stiffness matrices in 3D, East-West J. Numer. Math., 3(1):59-69, 1995.

[7] D. Leykekhman and B. Li, Weak discrete maximum principle of finite element methods in convex polyhedra, Math. Comp., 90:1-18, 2021.

[8] D. Leykekhman and B. Vexler, Finite element pointwise results on convex polyhedral domains, SIAM J Numer. Anal., 54(2):561-587, 2016.

[9] F. Natterer, Uber die punktweise Konvergenz finiter Elemente, Numer. Math. , 25:67-77, 1975.

[10] J.A. Nitsche, $L_{\infty}$ Convergence of Finite Element Approximations, Mathematical Aspects of Finite Element Methods. Lecture Notes in Math., vol. 606, pp. 261-274. Springer, Berlin (1977).

[11] R. Rannacher, Zur $L^{\infty}$-Konvergenz linearer finiter Elemente beim Dirichlet-Problem, Math.Zeitschrift, 149:69-77, 1976.

[12] R. Rannacher and R. Scott, Some optimal error estimates for piecewise linear finite element approximations, Math. Comp., 148:437-445, 1982.

[13] A.H. Schatz, A weak discrete maximum principle and stability of the finite element method in $L^{\infty}$ on the plane polygonal domains. I, Math. Comp., 34:77-91, 1980.

[14] A.H. Schatz, Pointwise error estimates and asymptotic error expansion inequalities for the finite element method on irregular grids: Part 1, Math. Comp., 67:877-899, 1998.

[15] A.H. Schatz and L.B. Wahlbin, On the quasi-optimality in $L_{\infty}$ of the $H^{1}$-projection into finite element spaces, Math. Comp., 157:1-22, 1982.

[16] R. Scott, Optimal $L_{\infty}$ estimates for the finite element method, Math. Comp., 30:681-697, 1976. 
[17] E. M. Stein, Singular integrals and differentiability properties of functions, Princeton Mathematical Series, No. 30, Princeton University Press, Princeton, N.J., 1970.

[18] J. Wang and R. Zhang, Maximum principles for $P_{1}$-conforming finite element approximation of quasilinear second order elliptic equations, SIAM J Numer. Anal., 50(2):626-642, 2012.

School of Mathematics and Statistics, Huazhong University of Science and Technology, WuHan 430074, China

Email address: huadong@hust.edu.cn

Department of Mathematics, City University of Hong Kong, Kowloon, Hong Kong, China

Email address: weifeqiu@cityu.edu.hk 